\title{
Editorial
}

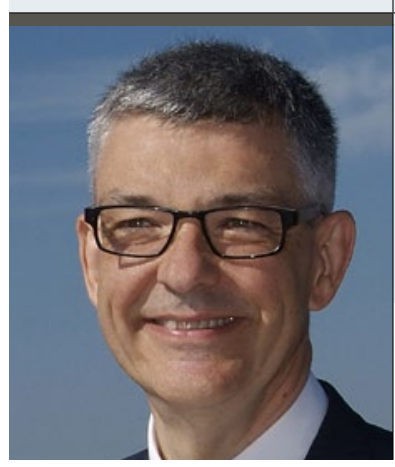

"Um das ganze Potenzial moderner Antipsychotika abzurufen,

sollten, häufiger als bislang in der klinischen Praxis üblich, frühe

Einstellungen auf Depot-Präparate vorgenommen werden."

\section{Bei Schizophrenie von Anfang an auf hohe Adhärenz setzen}

$\mathrm{N}$ ach Jahrzehnten mit zahlreichen pharmakotherapeutischen Innovationen sind die letzten Jahre in der klinischen Psychiatrie gekennzeichnet gewesen durch eine Konsolidierung des Erreichten. Wichtig ist hierbei die Frage, wie die im Spontanverlauf bekannten chronischen Verläufe psychiatrischer Erkrankungen durch den klugen Einsatz der uns bekannten Substanzen grundsätzlich günstiger gestaltet werden können. Zur Behandlung chronischer, therapieresistenter depressiver Erkrankungen informiert ein CME-Artikel in dieser Ausgabe (Seite 36).

\section{Hohes Risiko für Chronifizierung}

Schizophrene Erkrankungen sind charakterisiert durch das hohe Risiko nach der Erstmanifestation in einen zunehmend chronifizierenden Verlauf überzugehen, der mit strukturellen Veränderungen des Gehirns und typischen, progredienten kognitiven Störungen einhergeht („dementia praecox“ in der Originaldiktion Kraepelins). Das hohe Stigma mit dem Psychosen aus dem schizophrenen Formenkreis auch heute noch assoziiert sind, resultiert nicht zuletzt aus der Erkenntnis, dass die Schizophrenie irreversible Einschränkungen der Lebensqualität und der sozialen wie beruflichen Integrierbarkeit mit sich bringen könnte.

\section{Depot-Präparate unterstützen Adhärenz}

In der Therapie der Schizophrenie sind die ersten Monate nach korrekter Diagnosestellung und Anbehandlung von hoher Bedeutung. Gelingt es dem Patienten, eine Therapie mit Antipsychotika mit hoher Adhärenz durchzuführen, so sind Rückfälle seltener und die Chance auf eine psychosoziale Reintegration deutlich erhöht [1]. Nach Erstmanifestation einer Schizophrenie können bereits sehr kurze Zeitspannen reduzierter Adhärenz zu einer Exazerbation von Positivsymptomen führen [2]. Aus diesem Grund ist die Gabe eines modernen DepotAntipsychotikums der Gabe desselben Antipsychotikums in oraler Form bei Erstmanifestationen überlegen [3]. Die Ergebnisse der Studie von Subotnik et al. waren überraschend deutlich. In der Gruppe der oral medizierten (und bezüglich der Studie adhärenten!) Patienten hatten 50 \% nach einem Jahr ein Rezidiv: ein für ein solches Prozedere zu erwartendes Resultat. In der mittels Depot mit derselben Substanz (hier: Risperidon) behandelten Patienten hatten nach einem Jahr nur $8 \%$ ein Rezidiv. Dieses Ergebnis verdeutlicht, dass die frühe Gabe eines Depot-Antipsychotikums möglich ist und zu einer hohen Stabilität im Rehabilitationsprozess nach Erstmanifestation führen kann. Die besondere Empfindsamkeit für Rückfälle, die in den ersten Monaten nach Erstmanifestation bei Patientinnen und Patienten mit Schizophrenie besteht, sollte dazu führen, dass häufiger als bislang in der klinischen Praxis üblich, frühe Einstellungen auf Depot-Präparate vorgenommen werden, um das ganze Potenzial moderner Antipsychotika abzurufen.

Eine anregende Lektüre dieser Ausgabe von „DNP - Der Neurologe \& Psychiater" wünscht Ihnen,

Ihr

Andreas Erfurth

1. Fleischhacker WW, Allen C, Erfurth A et al. Therapieadhärenz bei Schizophrenie-Patienten. Psychiatrie und Psychotherapie 2011; 7 (3): 98-109

2. Subotnik KL, Nuechterlein KH, Ventura J et al. Risperidone nonadherence and return of positive symptoms in the early course of schizophrenia. Am J Psychiatry 2011; 168 (3): $286-92$

3. Subotnik KL, Casaus LR, Ventura J et al. Long-Acting Injectable Risperidone for Relapse Prevention and Control of Breakthrough Symptoms After a Recent First Episode of Schizophrenia. A Randomized Clinical Trial. JAMA Psychiatry 2015; 72 (8): 822-9 